COMMUNICATIONS IN

ANALYSIS AND GEOMETRY

Volume 11, Number 2, 223-233, 2003

\title{
Closed Geodesics on Oval Surfaces and Pattern Formation
}

\author{
C. E. Garza-Hume and P. Padilla
}

We study a singularly perturbed semilinear elliptic partial differential equation with a bistable potential on an oval surface. We show that the transition region of minimizers of the associated functional with a suitable constraint converges in the sense of varifolds to a minimal closed geodesic on the surface.

\section{Introduction.}

We consider the equation

$$
-\epsilon^{2} \Delta u+W^{\prime}(u)=0
$$

on a smooth oval surface $M$, where $\Delta$ represents the Laplace-Beltrami operator and $W$ is a nonlinear term to be specified later. Equation (1) arises in many contexts, for instance in materials science, superconductivity, population dynamics and pattern formation (see for instance [13] and references therein.)

Depending on the context, $u$ might represent an order parameter, concentration of a chemical, population density, etc. and is usually assumed to be defined on a two- or three-dimensional domain $\Omega$. Equation (1) is normally supplemented with a zero flux or homogeneous Neumann boundary condition

$$
\frac{\partial u}{\partial \nu}=0 \text { on } \partial \Omega
$$

where $\nu$ represents the outer normal to $\Omega$. For the nonlinear term $W$, a very important case is given by a bistable potential. In particular the AllenCahn-Hilliard equation and the Ginzburg-Landau model belong to this class. It would be impossible to list all the relevant works in this area, which has been actively studied for already several decades, both from the theoretical and the applied points of view. We refer the reader to [8] and [10] and the bibliography therein for a more comprehensive account. 
In this work we are interested in equation (1) on an oval surface. From the pattern formation or morphological perspectives it is more natural to consider equation (1) on a surface and not on subsets of the plane for various reasons. Some organisms, for example radiolarians, exhibit almost spherical symmetry. Pattern formation during the first stages of the embryo's development (up to the formation of the morula) may be considered to be taking place on the sphere. In [7] a similar equation was considered for a prototypical reduction of the Gierer-Meinhardt system, and, for $\epsilon \rightarrow 0$ an underlying geometrical problem, sphere-packing, was identified as the mechanism determining the behaviour of the limiting solutions. In [16] a problem on the sphere related to spiral waves is considered. Here to fix ideas we will take the bistable potential

$$
W(u)=\left(1-u^{2}\right)^{2},
$$

although this specific form is not needed as long as $W$ is bistable and symmetric (see assumption $\mathbf{A}$ in section 2.)

For $\epsilon \rightarrow 0$ we establish the behaviour of nontrivial minima. Again there is an underlying geometrical problem, this time related to a shortest closed geodesic on $M$. More specifically, we show that when $\epsilon \rightarrow 0$, nontrivial minimizers of the corresponding energy (with a suitable constraint) have a transition layer located at the shortest closed geodesic. This is not surprising if one considers the corresponding variational problem. Finding solutions of (1) is equivalent to finding critical points of the functional

$$
E_{\epsilon}(u)=\int_{M}\left(\frac{\epsilon}{2}|\nabla u|^{2}+\frac{1}{\epsilon} W(u)\right) d S,
$$

in a suitable function space.

For $\epsilon \rightarrow 0$, functions $u$ with uniformly bounded energy $E_{\epsilon}(u)<E_{0}$, can be proved to be close to \pm 1 in most of the domain, except for a transition curve (a codimension-1 hypersurface in the general case). Moreover,

$$
E_{\epsilon}(u) \simeq \text { length of the transition region, }
$$

that is, up to higher order terms the energy is proportional to the length of the transition.

Since the pioneering work of DeGiorgi much has been done to establish properties of $E$ rigorously (see for instance [14]). In particular in [8], [10] several convergence results for the transition layer are established, for a particular case in the first work and for the general case in the second. Roughly speaking, this result allows us to locate the interface and to handle it as a rectifiable curve. 
Once this is accomplished, the proof follows from a classical result in differential geometry due to Birkhoff that guarantees the existence of a closed geodesic on a surface diffeomorphic to the sphere.

We present here the basic idea of the proof using this geometrical fact. Consider an oval surface, $M$, that is, a closed and compact surface which encloses a strictly convex domain in $\mathbf{R}^{3}$. We will also require $M$ to be smooth. A well-known result stated by Poincaré in [9] and studied in detail later on in [3] is the following:

Proposition 1. Suppose that $\gamma$ is a closed curve on $M$ that under the Gauss map, $g$, divides the unit sphere in two parts of equal measure. Assume further that among all the curves satisfying the above conditions, $\gamma$ has minimal length. Then $\gamma$ is a closed geodesic.

This suggests a natural constraint. So the main point consists in minimizing $E_{\epsilon}$ among functions $u$ such that

$$
\int_{S^{2}} u\left(g^{-1}(y)\right) d \tilde{\sigma}=0 \text { and } E_{\epsilon}<E_{0}
$$

For $\epsilon$ small, since the interface is localized and close to the zero level set of $u$ and $u$ is close to either +1 or -1 elsewhere, one expects this restriction to be equivalent to imposing the condition that it divides $S^{2}$, under the Gauss map, into two regions of equal area. Then we can show that the Lagrange multiplier introduced by condition (2) goes to zero, so that critical points for the constrained problem are still approximate solutions of (1).

Using the results of [8] and the result by Birkhoff mentioned above we show that the interface converges, as $\epsilon \rightarrow 0$, to a minimal closed geodesic. For a precise statement of the result see theorem 3.

Similar problems have been analysed using $\Gamma$-convergence (see [1]) but we decided to use the results of [8] for two reasons. First, $\Gamma$-convergence only applies to minima and the results of [8] apply to general critical points (see section 4). Second, the approach in [8] allows us to obtain a multiplicity result for certain nonconvex domains constructed by "glueing together" convex ones (see the end of section 3). Moreover, the results of Ljusternik and Schnirelman guarantee the existence of closed geodesics that are not minima. In this case the general framework of [8] would be needed.

In the next section we recall some facts about equation (1) and its variational structure. We also present the results we need from [8]. In section 3 we prove the existence of nontrivial approximate solutions of equation (1) by minimizing $E_{\epsilon}$ subject to (2) and establish the geometric properties of 
the solution when $\epsilon \rightarrow 0$. Finally, section 4 is devoted to the discussion of related open problems.

\section{Preliminary Results.}

In this section we introduce the necessary notation and quote some results from [8]. We require that $W$ satisfies the following assumptions:

\section{Assumptions}

A : The function $W: \mathbf{R} \rightarrow[0, \infty)$ is $C^{3}$ and $W( \pm 1)=0$. For some $\mu \in$ $(-1,1), W^{\prime}<0$ on $(\mu, 1)$ and $W^{\prime}>0$ on $(-1, \mu)$. For some $\alpha \in(0,1)$ and $\kappa>0, W^{\prime \prime} \geq \kappa$ for all $|x| \geq \alpha$.

This is to ensure that $W$ is $W$-shaped with non-degenerate minima at \pm 1 and local maximum at $\mu$.

B : $U \subset \mathbf{R}^{n}$ is a bounded open set with Lipschitz boundary $\partial U$. A sequence of $C^{3}(U)$ functions $\left\{u^{i}\right\}_{i=1}^{\infty}$ satisfies

$$
\epsilon_{i} \Delta u^{i}=\epsilon_{i}^{-1} W^{\prime}\left(u^{i}\right)-\lambda_{i}
$$

on $U$. Here, $\lim _{i \rightarrow \infty} \epsilon_{i}=0$, and we assume there exist $c_{0}, \lambda_{0}$ and $E_{0}$ such that $\sup _{U}\left|u^{i}\right| \leq c_{0},\left|\lambda_{i}\right| \leq \lambda_{0}$ and

$$
\int_{U} \frac{\epsilon_{i}\left|\nabla u^{i}\right|^{2}}{2}+\frac{W\left(u^{i}\right)}{\epsilon_{i}} \leq E_{0}
$$

for all $i$.

Let

$$
\phi(s)=\int_{0}^{s} \sqrt{W(s) / 2} d s
$$

and define new functions

$$
w^{i}=\phi \circ u^{i}
$$

for each $i$.

Let $G(n, n-1)$ be the Grassman manifold of unoriented $(n-1)$-dimensional planes in $\mathbf{R}^{n}$. We regard $S \in G(n, n-1)$ as the orthogonal projection of $\mathbf{R}^{n}$ onto $S$. We say $V$ is an $(n-1)$-dimensional varifold in $U \subset \mathbf{R}^{n}$ if $V$ is a Radon measure on $G_{n-1}(U)=U \times G(n, n-1)$. Let $V_{n-1}(U)$ denote the set of all $(n-1)$-dimensional varifolds in $U$. Convergence in the varifold sense means convergence in the usual sense of measures. For $V \in V_{n-1}(U)$ we let the weight $\|V\|$ be the Radon measure in $U$ defined by

$$
\|V\|(A)=V(\{(x, S) \mid x \in A, S \in G(n, n-1)\})
$$


for each Borel set $A \subset U$. If $M$ is a $(n-1)$-rectifiable subset of $U$ we define $v(M) \in V_{n-1}(U)$ by

$$
v(M)(E)=\mathcal{H}^{n-1}\left(\left\{x \in U \mid\left(x, \operatorname{Tan}^{n-1}\left(\mathcal{H}^{n-1}\left\lfloor_{M}, x\right)\right) \in E\right\}\right)\right.
$$

for each Borel set $E \in G_{n-1}(U)$, where $\operatorname{Tan}^{n-1}\left(\mathcal{H}^{n-1}\lfloor M, x)\right.$ is the approximate tangent plane to $M$ at $x$ and so exists for $\mathcal{H}^{n-1}$ a.e. $x \in M$.

We associate to each function $w^{i}$ a varifold $V^{i}$ ([5], [12]) defined as follows:

$$
V^{i}(A)=\int_{-\infty}^{\infty} v\left(\left\{w^{i}=t\right\}\right)(A) d t
$$

for each Borel set $A \subset G_{n-1}(U)$.

By the compactness theorem for BV functions, there exists an a.e. pointwise limit $w^{\infty}$. Let $\phi^{-1}$ be the inverse of $\phi$ and define

$$
u^{\infty}=\phi^{-1}\left(w^{\infty}\right) \text {. }
$$

$u^{\infty}= \pm 1$ a.e. on $U$ and the sets $\left\{u^{\infty}= \pm 1\right\}$ have finite perimeter in $U$.

In [8] the following theorem is proved:

Theorem 1. Let $V^{i}$ be the varifold associated with $u^{i}$ (via $\left.w^{i}\right)$. On passing to a subsequence we can assume

$$
\lambda_{i} \rightarrow \lambda_{\infty}, \quad u^{i} \rightarrow u^{\infty} \text { a.e., } \quad V^{i} \rightarrow V \text { in the varifold sense. }
$$

Moreover,

(1) For each $\phi \in C_{c}(U)$,

$$
\begin{aligned}
\|V\|(\phi) & =\lim _{i \rightarrow \infty} \int \phi \frac{\epsilon_{i}\left|\nabla u^{i}\right|^{2}}{2}=\lim _{i \rightarrow \infty} \int \phi \frac{W\left(u^{i}\right)}{\epsilon_{i}} \\
& =\lim _{i \rightarrow \infty} \int \phi\left|\nabla w^{i}\right| .
\end{aligned}
$$

(2) $\operatorname{supp}\left\|\partial\left\{u^{\infty}=1\right\}\right\| \subset \operatorname{supp}\|V\|$, and $\left\{u^{i}\right\}$ converges locally uniformly to \pm 1 in $U \backslash \operatorname{supp}\|V\|$, where $\partial$ denotes the reduced boundary.

(3) For each $\tilde{U} \subset \subset U$ and $0<b<1,\left\{\left|u^{i}\right| \leq 1-b\right\} \cap \tilde{U}$ converges to $\tilde{U} \cap \operatorname{supp}\|V\|$ in the Hausdorff distance sense.

(4) $\sigma^{-1} V$ is an integral varifold. Moreover, the density $\theta(x)=\sigma N(x)$ of $V$ satisfies

$$
N(x)=\left\{\begin{array}{lll}
\text { odd } & \mathcal{H}^{n-1} \text { a.e. } x \text { in } M^{\infty} \\
\text { even } & \mathcal{H}^{n-1} \text { a.e. } x \in \operatorname{supp}\|V\| \backslash M^{\infty}
\end{array}\right.
$$


where $M^{\infty}$ is the reduced boundary of $\left\{u^{\infty}=1\right\}$ and

$$
\sigma=\int_{-1}^{1} \sqrt{W(s) / 2} d s
$$

(5) The generalized mean curvature $H$ of $V$ is given by

$$
H(x)= \begin{cases}\frac{\lambda_{\infty}}{\theta(x)} \nu^{\infty}(x) & \mathcal{H}^{n-1} \text { a.e. in } M^{\infty} \\ 0 & \mathcal{H}^{n-1} \text { a.e. } x \in \operatorname{supp}\|V\| \backslash M^{\infty}\end{cases}
$$

where $\nu^{\infty}$ is the inward normal for $M^{\infty}$.

Theorem 2 is also proved in [8].

Theorem 2. In addition to assumptions $\mathbf{A}$ and $\mathbf{B}$ suppose $\left\{u^{i}\right\}$ are locally energy minimizing on $\tilde{U} \subset \subset U$ for $E_{\epsilon_{i}}$ (with or without volume constraint). Then $N(x)=1, \mathcal{H}^{n-1}$ a.e. on $\tilde{U} \cap \operatorname{supp}\|V\|$. The set $\partial\left\{u^{\infty}=1\right\}$ on $\tilde{U}$ has constant mean curvature $\frac{\lambda_{\infty}}{\sigma} \nu^{\infty}$ and no energy loss occurs on $\tilde{U}$.

These results are proved for subsets of $\mathbf{R}^{n}$ but all the proofs are based on local estimates and therefore theorems 1 and 2 can be extended to our setting by taking local coordinates. Also, our equation requires a slightly more general result, when $\lambda_{i}$ might depend on $x$ but this has been recently proved in [15].

\section{Existence and properties of solutions.}

We will characterize the behaviour of minimal solutions of the following problem when $\epsilon \rightarrow 0$ :

$$
\inf E_{\epsilon}(u)=\inf \int_{M}\left(\frac{\epsilon|\nabla u|^{2}}{2}+\frac{W(u)}{\epsilon}\right) d \sigma
$$

for all $u \in H^{1}(M)$, subject to the restriction

$$
G(u)=\int_{M} u(y) f(y) d \sigma=0
$$

where $f(y)$ is the determinant of the Jacobian of the transformation from $S^{2}$ to $M$ and can be chosen to be positive (since for an oval surface the Gauss 
map is a diffeomorphism.) In fact this factor is just the Gaussian curvature at $y$. The associated Euler-Lagrange equation is

$$
\epsilon_{i} \Delta u^{+} \frac{1}{\epsilon_{i}} W(u)+\lambda_{i} f=0,
$$

where $\lambda_{i}$ is the corresponding Lagrange multiplier. Our result is the following:

Theorem 3. When $\epsilon \rightarrow 0$ the varifolds $V^{i}$ associated to minimal solutions of (3), (4) converge (up to a subsequence) in the sense of varifolds to a closed geodesic of minimal length, $\gamma_{0}$, on $M$. In fact, as in point 3 in theorem 1, for each compact $\tilde{U} \subset M$ and $0<b<1,\left\{\left|u^{i}\right| \leq 1-b\right\} \cap \tilde{U}$ converges to $\tilde{U} \cap \operatorname{supp}\left\|V_{\gamma_{0}}\right\|$ in the Hausdorff distance sense, where $V_{\gamma_{0}}$ is the varifold associated with $\gamma_{0}$.

Proof: The existence of a minimizer for a fixed $\epsilon>0$ is immediate. Indeed it is a standard fact that this functional satisfies the Palais-Smale condition (see [11]) and since the restriction defines a closed linear subspace the infimum is achieved. Also, the minimum cannot be a constant for $\epsilon$ sufficiently small since the only constant satisfying the restriction is $u_{0} \equiv 0$, but

$$
E_{\epsilon}\left(u_{0}\right) \geq \frac{\operatorname{Vol}(M)}{\epsilon} W(0) \rightarrow \infty
$$

as $\epsilon \rightarrow 0$. On the other hand there are trial functions $\tilde{u}_{\epsilon}$ such that

$$
E_{\epsilon}\left(\tilde{u}_{\epsilon}\right)<E_{0}
$$

for some $E_{0}>0$. One way to construct such a function is to take the inverse image under the Gauss map of a smooth curve $\gamma \subset S^{2}$ dividing $S^{2}$ in two parts of equal area. Then $\Gamma=g^{-1}(\gamma)$ is a smooth curve on $M$. We can construct $\tilde{u}_{\epsilon}$ by locating a transition layer on $\Gamma$ (see [6] or [14] for details.)

We will now show that the Lagrange multiplier of the constrained problem tends to zero when $\epsilon \rightarrow 0$.

First observe that the restriction is natural in the following senses (see [3] lemma 1):

(a) every nontrivial closed simple smooth geodesic on $M$ satisfies the constraint

$$
\int_{\Sigma(C)} K d V=2 \pi
$$

where $K$ is the Gaussian curvature of $M$ and $\Sigma(C)$ denotes a simply connected subset of $M$ bounded by $C$. 
(b) every critical point in $\mathcal{C}$ of the arclength functional on $M$ with the constraint (3) is automatically a critical point of the unrestricted arclength functional.

Note that the constraint (6) is equivalent to the one used in proposition 1 since the integral of the Gaussian curvature over the whole domain is $4 \pi$.

Then it follows from the work by X. Chen ([4]) and from the fact that $M$ is regular that the Lagrange multipliers $\lambda_{i}$ corresponding to the EulerLagrange equation (5) are bounded so assumption B in section 2 is satisfied.

The rest of the proof is by contradiction. We know from a classical result by Birkhoff that there is a closed geodesic on the oval surface. We state the theorem for completeness:

Theorem 4. ([11], Thm. II.4.4 or [2]) On any compact surface $S$ in $\mathbf{R}^{3}$ which is $C^{3}$-diffeomorphic to the standard sphere, there exists a non-constant closed geodesic.

Suppose the sequence of minimizers does not concentrate on a minimal closed geodesic. Then by the convergence results (theorems 1 and 2) and standard approximation by Lipshitz functions of rectifiable varifolds (see [12]), we may identify the sequence $V^{i}$ with a sequence of Lipschitz curves $\gamma^{i}$ converging, in the Hausdorff sense, to the support of $V$ (see point 3 in theorem 1). Moreover, since $u_{\epsilon}$ is regular by elliptic regularity, we see using Sard's theorem that $u^{-1}(C)$ is a union of smooth closed curves for almost every $C$ and so $\gamma^{i}$ has to be a union of such curves too. Accordingly, we have:

$$
E_{\epsilon_{i}}\left(u^{i}\right)=A\left(\text { length of } \gamma^{i}\right)+o(\epsilon),
$$

where $A$ is a constant (independent of $\epsilon$ ). Let $\gamma_{0}$ be the geodesic given by Birkhoff's result (theorem 4). Construct a function $u_{0_{\epsilon}}$ with a transition at $\gamma_{0}$ in the way described above. It follows that $u_{0_{\epsilon}}$ is an admissible function. Moreover

$$
E_{\epsilon}\left(u_{0}\right)=A\left(\text { length of the transition layer for } u_{0}\right)+o(\epsilon)
$$

Then

$$
E_{\epsilon}\left(u_{0}\right)<E_{\epsilon}\left(u^{i}\right)
$$

for sufficiently small $\epsilon$ thus contradicting the minimality of $u^{i}$.

This proves theorem 3 .

It remains to observe that by the naturality (in the sense of (a) and (b) above) of constraint (3) implies by contradiction that the Lagrange multipliers $\lambda_{i}$ tend to zero as $i \rightarrow \infty$. 
We now show how the results of [8] enable us to localize the previous argument and give a multiplicity result in a specific example.

Consider the domain $\Omega=\Sigma_{1}^{0} \cup \Sigma_{2}^{0} \cup \Sigma_{3}$, where $\Sigma_{1}$ and $\Sigma_{2}$ are two overlapping ovals. The region of overlap is contained in a slab $\Lambda=\left\{a<x_{1}<b\right\}$, in this region $\Sigma_{1}$ and $\Sigma_{2}$ are smoothly connected by another surface $\Sigma_{3}$ and $\Sigma_{i}^{0}=\Sigma_{i} \backslash \Lambda$ (see figure 1$)$.

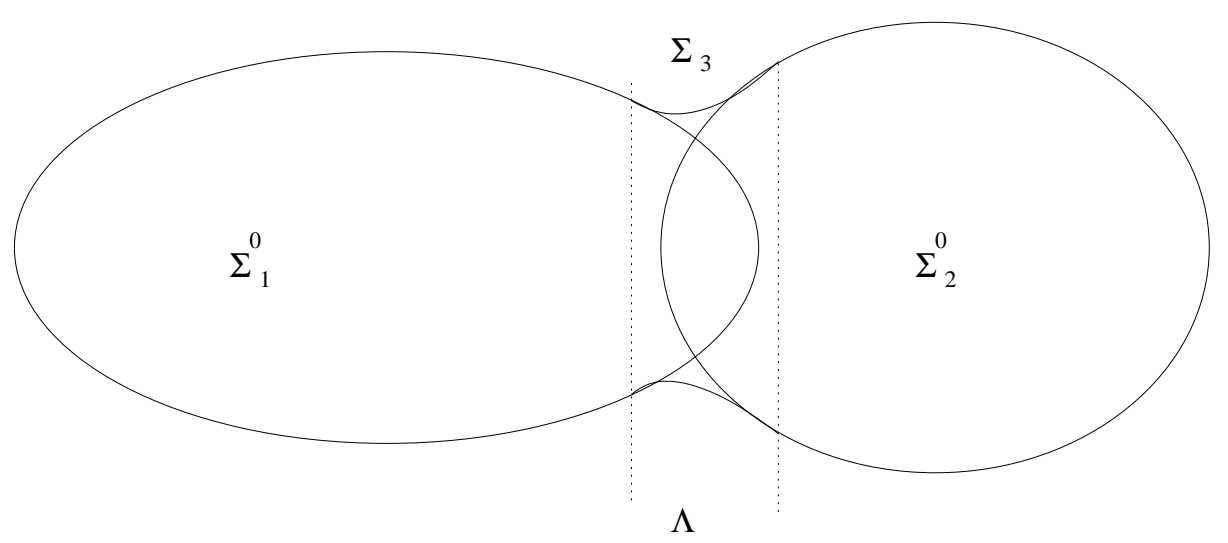

Figure 1: Region $\Omega$

We assume that the minimal geodesics $\gamma_{1}$ and $\gamma_{2}$ of $\Sigma_{2}$ and $\Sigma_{2}$ respectively do not intersect $\Lambda$. Then we will consider the same functional as before but with a different constraint. To do that we observe that there is a diffeomorphism $\phi$ from $\Sigma_{2}^{0} \cup \Sigma_{3}$ to $\Sigma_{1} \backslash \Sigma_{1}^{0}$. Then the Gauss map $g$ of $\Sigma_{1}$ composed with $\phi$ is well-defined in $\Omega: \tilde{g}=g \circ \phi=g(\phi(x)) \rightarrow S^{2}$. So we can apply the same procedure as before with the restriction

$$
\int_{S^{2}} u\left(\tilde{g}^{-1}(x)\right)=0
$$

and imposing the additional pointwise constraint $1-u(x)<\delta$ in $\Sigma_{3} \cup \Sigma_{2}^{0}$ to ensure that the transition lies in $\Sigma_{1}^{0}$. So we obtain, as $\epsilon \rightarrow 0$, a minimal geodesic on $\Sigma_{1}^{0}$. By the convergence results in [8] we know that $u_{\epsilon} \rightarrow \pm 1$ uniformly away from the transition so the pointwise constraint is automatically met for $\epsilon$ sufficiently small. Clearly the same procedure can be repeated to obtain a geodesic on $\Sigma_{2}$. 


\section{Conclusions and open problems.}

There are two aspects that we would like to emphasize. The first is that the approach used here could in principle be used to determine multiplicity results for closed geodesics on manifolds. It would provide an alternative way of proving the results of Ljusternik and Schnirelman, namely the existence of three geometrically distinct non-self-intersecting closed geodesics on any surface of genus 0 .

It would be natural to expect that multiplicity results for equation (1) would give corresponding results for closed geodesics. However, there are several points to consider. Ljusternik and Schnirelman's results are valid for general manifolds, not necessarily oval so the first question is whether our approach goes through in this case.

Also, we used the minimality of the sequence of solutions in order to prove that concentration has to take place on a geodesic. Notice that for multiple geodesics one would have to deal with general critical points of $E$.

Finally we observe that our approach generalizes directly to oval manifolds in higher dimensions and codimension-one minimal submanifolds.

\section{Acknowledgements.}

This work was supported in part by Conacyt Group Project G25427-E and project 34203-E. We thank Ana Pérez Arteaga for computational support.

\section{References.}

[1] Ambrosio, L., Fusco, N., Pallara, D. Functions of bounded variation and free discontinuity problems, Oxford Mathematical Monographs, The Clarendon Press, Oxford University Press, New York 2000.

[2] Birkhoff, G. D., Dynamical systems with two degrees of freedom, Trans. Amer. Math. Soc. 18 (1917) pp. 199-300.

[3] Berger, M. S., Bombieri E., On Poincaré's Isoperimetric Problem for Simple Closed Geodesics, J. Functional An., 42, 274-298 (1981).

[4] Chen, X., Global asymptotic limit of solutions of the Cahn-Hilliard equation, J. Diff. Geom. 44 no.2, 262-311 (1992).

[5] Federer, Geometric Measure Theory, Die Grundlehren der mathematischen Wissenschaften, Band 153, Springer-Verlag, New York (1969). 
[6] Flores, G., Padilla, P., Tonegawa, Y., Higher energy solutions in the theory of phase transitions: a variational approach, Journal of Differential Equations 169, 190-207 (2001).

[7] Garza-Hume. C. E., Padilla, P., A pattern formation problem on the sphere. Preprint.

[8] J. E. Hutchinson, Y. Tonegawa, Convergence of phase interfaces inthe van der Waals-Cahn-Hilliard theory, Calc. Var. 10, pp.49-84 (2000).

[9] Poincaré, H., Sur les lignes géodésiques des surfaces convexes, Trans. Amer. Math. Soc. 6 (1905), 237-274.

[10] Padilla, P. and Tonegawa, Y., On the convergence of stable phase transitions, CPAM LI, 551-579, 1998.

[11] Struwe, M., Variational Methods, Springer-Verlag 1990.

[12] Simon, L., Lectures on Geometric Measure Theory, Proc. Centre Math. Anal. Austral. Nat. Univ. 3 (1983).

[13] Smoller, J., Shock waves and reaction-diffusion equations, Springer Verlag, Berlin 1992.

[14] Sternberg, P., Zumbrun, K. Connectivity of phase boundaries in strictly convex domains, Arch. Rational Mech. Anal. 141 (1998), no. 4, 375-400.

[15] Tonegawa, Y., Phase Field Model with a Variable Chemical Potential, Hokkaido Univ. Preprint Series in Math., series 509, Dec. 2000, Japan.

[16] Yagisita, H., Mimura, M., Yamada, M., Spiral wave behaviors in an excitable reaction-diffusion system on a sphere, Physica D, 124(1-3), pp.126-136, 1998.

Institute of Applied Mathematics (IIMAS), UNAM

Mexico City, Mexico

E-mail: clara@uxmym1.iimas.unam.mx AND pablo@uxmym1.iimas.unam.mx

Received JANuARY 16, 2002. 